\title{
Quantitative real-time PCR for gene dosage determinations in microdeletion genotypes
}

\author{
Jonathan Covault, Christine Abreu, Henry Kranzler, and Cheryl Oncken \\ University of Connecticut School of Medicine, Farmington, CT, USA
}

BioTechniques 35:594-598 (September 2003)

Phase I and II metabolic enzymes are involved in the metabolism and elimination of a variety of compounds, including potentially harmful components of tobacco smoke. Null deletion mutations of genes encoding two phase II enzymes, glutathione S-transferase $\theta$ 1 (GSTT1, 22q11.2), and glutathione S-transferase $\mu 1$ (GSTM1, 1p13.3) are common, with up to $20 \%$ and $50 \%$ of subjects, respectively, homozygous for deletions at each locus (1). Subjects that are homozygous for deletions of either GSTM1 or GSTT1 have been reported to be at an increased risk for several cancers (2-6) and other smoking-related illnesses (7). The prevalence of the heterozygous condition for these loci (i.e., deletion on a single chromosome) has not typically been reported because the nonquantitative end point PCR assays used to investigate these deletions do not differentiate the homozygous-present from heterozygote genotypes (1-7).

Wiebel et al. (8), using a gel-based semiquantitative PCR assay of the GSTT1 deletion, observed that in vitro GSTT1 enzyme activity was quantitatively related to gene dosage in a large pedigree. This observation is consistent with measurements of GSTT1 enzyme activity in larger samples that show subjects clustering into three groups: high conjugators, low conjugators, and nonconjugators (9). Together, these results suggest that the ability to differentiate the homozygous-present genotype (high activity) from the heterozygote genotype (low activity) might be important for studies that correlate clinical phenotypes with GSTTI and GSTM1 genotypes (e.g., for assessing health outcomes related to a variety of environmental exposures including tobacco smoke). Here we report the use of a TaqMan ${ }^{\circledR}$ (Applied Biosystems, Foster City, CA, USA)-based quantitative PCR assay to differentiate
0,1 , or 2 copies of the GSTT1 or GSTM1 genes. The method can be readily applied to genotype other similar gene deletions and should be useful for the quantitative investigation of a variety of deletion or trisomy genetic conditions. This method has been previously reported to be capable of identifying transgene copy numbers in transformed plants (10).

Quantitative real-time PCR was carried out using the TaqMan (5'-nuclease) assay system (11-13) with signal from a GSTT1- or GSTM1-specific probe that is normalized to the signal for a reference autosomal gene [cannabis receptor $(C N 1)$ or $\beta$-2-microglobulin $(B 2 M)$ ] using DNA samples from 270 Caucasian subjects of European-American decent. The DNA samples used in this study were originally obtained as controls for genetic studies of substance dependence. All subjects provided IRB-approved written informed consent at the time of blood sample collection for the genetic analysis of purified DNA.

TaqMan probes and primers were designed using the Primer Express ${ }^{\circledR}$ software package (Applied Biosystems) and genomic sequences from the November 2002 freeze of the human genome database (http://www.genome. ucsc.edu). We compared primer and probe sequences for target and reference genes to avoid cross-hybridization between oligonucleotides. A Basic Local Alignment Search Tool (BLAST; National Center for Biotechnology Information, Bethesda, MD, USA) was used to eliminate the selection of products with significant sequence similarity at other genetic loci. Finally, because the utility of the assay depends on similar efficiencies of amplification of the target and reference genes, we verified that the slope of $\Delta$ cycle threshold $\left(\Delta \mathrm{C}_{\mathrm{t}}\right)$ versus input DNA was $<0.1$ over a 100 -fold genomic DNA dilution series.

To assay GSTT1 copy number relative to the reference gene $C N 1$, triplicate PCRs $(20 \mu \mathrm{L})$ containing $2 \mathrm{ng}$ genomic DNA, $300 \mu \mathrm{m}$ each GSTT1 primers (forward, 5'-CTGTGGTCCCCAAATCAGATG- $3^{\prime}$ and reverse, $5^{\prime}$ TTGGGTCGGCCTTCGAA- $3^{\prime}$ ), CN1 primers (forward, 5'-CACAGCCATCGACAGGTACATATC-3' and reverse, 5'-CCTTGGGCCTGGTGACAA-3'), and fluorescent oligonucleotide probes specific for each product containing a 3'-quencher [TaqMan Minor Groove Binder (MGB); GSTT1, FAM-TGCCCTCACAACCAT-MGB $(60 \mathrm{nM})$ and CN1, VIC-CCTGGCCTATAAGAGGMGB $(120 \mathrm{nM})]$ were prepared in $1 \times$ TaqMan Universal PCR Master Mix (Applied Biosystems). Thermal cycling was carried out in 96-well plates with realtime fluorescence detection using an $\mathrm{ABI}$ PRISM $^{\circledR} 7700$ thermal cycler (Applied Biosystems). The samples were incubated for $10 \mathrm{~min}$ at $95^{\circ} \mathrm{C}$ and then cycled 35 times at $95^{\circ} \mathrm{C}$ for $20 \mathrm{~s}$, followed by $60^{\circ} \mathrm{C}$ for $60 \mathrm{~s}$. To assay GSTM1 copy number relative to the reference gene $B 2 M$, we used $300 \mu \mathrm{M}$ each gene-specific GSTM1 primers (forward, 5'-AAGATTCGTGTGGACATTTTGGA- $3^{\prime}$ and reverse, 5'-TCTGGATTGTAGCAGATCATGCC-3'), B2M primers (forward, 5' TTGTTTCACTGTCCTGAGGACTATTTAT- $3^{\prime}$ and reverse, 5'-ATGTTACTCTGTCAATGTTCTCCACAT-3'), and fluorescent product-specific probes GSTM1 (FAM-CAGACCATGGACAACCA-MGB; $120 \mathrm{nM}$ ) and B2M (VICCTCTAACATGATA ACCCTCACMGB; $30 \mathrm{nM}$ ) using identical thermal cycling conditions as for GSTT1 primers.

Log-normalized change in fluorescence intensity versus cycle number amplification plots was generated using the ABI PRISM 7700 Sequence Detection Systems (SDS) 1.7 software (Applied Biosystems), and a fluorescence intensity threshold was manually selected in the middle portion of the exponential PCR amplification phase for each assay plate. The cycle number at which the change in fluorescence intensity reaches this threshold is then automatically identified by the software for FAM (GSTT1 or GSTM1) and VIC (CN1 or B2M) fluorescence for each well. Figure 1 illustrates representative TaqMan amplification plots for three samples, each in triplicate, using this assay. Figure 1A contains the amplification profiles for the VIC-B2M reference gene 
product, and Figure 1B contains the amplification profiles of the FAM-GSTM1 product. The reference gene amplification curves overlap, which indicates nearly equal amplification efficiency and input DNA amount for each well. In contrast, the GSTT1 curves separate into three distinct groups consistent with $2 \mathrm{~N}$-homozygote, $1 \mathrm{~N}$-heterozygote, and null genotypes for GSTM1. A pattern of poorly clustered triplicates without a sharp exponential rise are typical of TaqMan reactions for samples with very low or no input product targets. The increase in fluorescence seen in late cycles for the null genotypes (Figure 1B) represents nonspecific products generated under the high $\mathrm{Mg}$ concentrations used in the TaqMan buffer system to drive high-efficiency amplification. Samples that do not demonstrate a sharp exponential rise in the reference gene (VIC) fluorescence before cycle 26 were considered failed amplifications and repeated. The average $\Delta \mathrm{C}_{\mathrm{t}}$ (e.g., CN1-GSTT1) was calculated for each triplicate from the $C_{t}$ values for each well. Results for each plate were plotted in a $\Delta \mathrm{C}_{\mathrm{t}}$ versus $\Delta \mathrm{C}_{\mathrm{t}}$ graph, generating three distinct clusters of $\Delta \mathrm{C}_{\mathrm{t}}$ samples. $\Delta \mathrm{C}_{\mathrm{t}}$ values were scaled for each assay plate to give an average $\Delta \mathrm{C}_{\mathrm{t}}$ of 1 for heterozygotes (intermediate $\Delta \mathrm{C}_{\mathrm{t}}$ cluster) to plot results from several plates on a common graph. For GSTT1, the raw heterozygote $\Delta \mathrm{C}_{\mathrm{t}}$ mean $(\overline{\mathrm{x}} \pm \mathrm{SD})$ for nine plates was $-0.33 \pm 1.29$. To assay small sets of unknown samples, a set of known DNAs can be included with each plate to identify the $\Delta \mathrm{C}_{\mathrm{t}}$ cluster values to facilitate genotype assignment.

Figure $2 \mathrm{~A}$ is a dot plot of $\Delta \mathrm{C}_{\mathrm{t}}$ values using this method for GSTT1 deletion genotyping of personally de-identified genomic DNA from 270 healthy control European-American subjects from Connecticut. Heterozygous GSTT1 deletion and homozygous GSTT1-present DNA samples cluster in two groups with a difference in mean $\Delta \mathrm{C}_{\mathrm{t}}$ of 0.9 , which is consistent with one versus two copies of GSTT1 target because during the exponential phase, there is approximately a doubling of target sequence for each PCR cycle. Heterozygote samples (1N copy number) reach cycle threshold one cycle later on average compared with homozygous-present samples ( $2 \mathrm{~N}$ copy number) (Figure 1B, green vs. red curves), while the average cycle threshold for the reference gene is unchanged. Subjects with the deletion of both GSTTI alleles fail to generate an increase in FAM fluorescence until later cycles, if at all (e.g., Figure 1B, blue curves), despite normal amplification of the reference gene target, producing $\Delta \mathrm{C}_{\mathrm{t}}$ s from -4 to -12. The mean $(\overline{\mathrm{x}} \pm \mathrm{SD})$-normalized $\Delta \mathrm{C}_{\mathrm{t}}$ values for the group of 270 subjects examined were homozygous-present with $1.9 \pm 0.14$; heterozygotes with $1.0 \pm 0.15$;

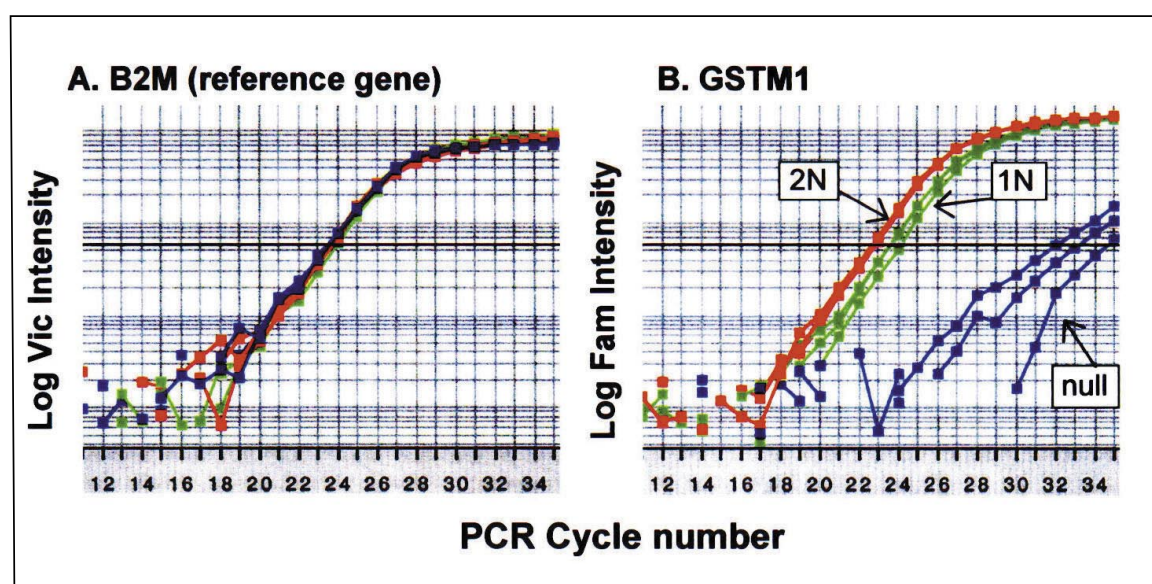

Figure 1. Log fluorescence intensity change versus PCR cycle number for the TaqMan quantitative real-time PCR assay. (A) The $B 2 M$ reference gene (VIC probe) amplification profiles from triplicate wells for three DNA samples, illustrating nearly identical cycle thresholds, with average $C_{t} s$ of 23.4 , 23.6, and 23.3 for red, green, and blue tracings, respectively. (B) The corresponding same-well GSTM1 (FAM probe) amplification profiles, with average $\mathrm{C}_{\mathrm{t}} \mathrm{s}$ of $22.8,23.8$, and 33.4 for the red, green, and blue tracings. The corresponding $\Delta \mathrm{C}_{\mathrm{t}}$ values are $+0.64,-0.22$, and -10.1 , respectively, indicating a relative copy number of $2^{\Delta \mathrm{C}_{\mathrm{t}}}$ or $1.8,1$, and 0.001 for the three samples, which is consistent with $2 \mathrm{~N}$ homozygote (red), $1 \mathrm{~N}$ heterozygote (green), and null genotypes (blue). The dark horizontal line at the midpoint of exponential amplification indicates the threshold intensity value for this plate. and double deletions with $-10.4 \pm 3.9$. The frequencies for the three genotypes in this sample were $0.285,0.570$, and 0.144 , respectively, which are consistent with Hardy-Weinberg equilibrium for the observed frequency of the GSTT1 allele deletion of $0.43\left(\chi^{2}=3.9 ; P=0.14\right)$.

To assay GSTMI copy number, a second reference gene, $B 2 M$, was used to demonstrate that the assay could be used with alternative reference genes. Figure 2B shows a dot plot of $\Delta C_{t}$ values for GSTM1 deletion genotyping for the sample of European-American subjects examined for the GSTT1 deletion (Figure $2 \mathrm{~A}) . \Delta \mathrm{C}_{\mathrm{t}}$ values again clustered into three groups: homozygous-present with $\Delta \mathrm{C}_{\mathrm{t}}=1.90 \pm 0.15$; heterozygotes with $\Delta \mathrm{C}_{\mathrm{t}}=0.98 \pm 0.17$; and double deletions with $\Delta \mathrm{C}_{\mathrm{t}}=-9.1 \pm 2.3$. The frequencies for these three genotypes in the subject sample were $0.054,0.414$, and 0.532 , respectively, which are consistent with

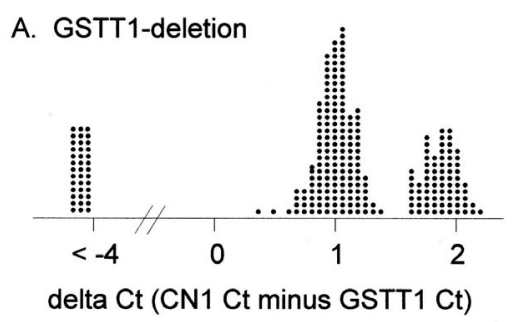

B. GSTM1-deletion

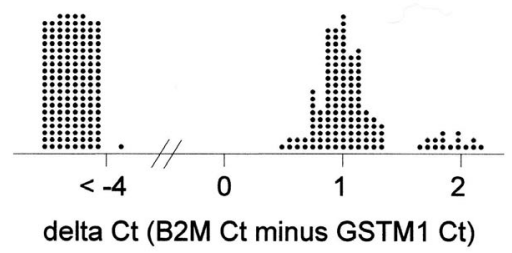

Figure 2. Pooled quantitative PCR data from nine assay plates. (A) Dot plot of the difference in cycle threshold number for GSTT1 or (B) GSTM1 relative to a reference gene amplified in the same well $(\mathrm{CN} 1$ for panel $\mathrm{A}$ or $\mathrm{B} 2 \mathrm{M}$ for panel B). Higher concentrations of target DNA generate threshold fluorescence signal after fewer cycles, and lower $\Delta C_{t}$ values (reference gene $C_{t}$ - target gene $C_{t}$ ) indicate low levels of GSTT1 or GSTM1 relative to the reference gene. Heterozygous subjects ( 1 allele deleted) have a $\Delta \mathrm{C}_{\mathrm{t}}$ average of 1; homozygous-present subjects have $\Delta \mathrm{C}_{\mathrm{t}}$ s of 1.6-2.2; while homozygous deletion subjects have $\Delta C_{t}$ values in the -4 to -16 range. The frequency of deleted alleles was $43 \%$ for GSTT1 and 74\% for GSTM1 for this sample of European-American subjects. 
Hardy-Weinberg equilibrium for the observed frequency of GSTM1 allele deletion of $0.74\left(\chi^{2}=0.8 ; P=0.68\right)$. To validate our results, we also compared them with the currently used method of genotyping GSTT1 and GSTM1 null deletions. End point (35 cycles) PCR products from 10 subjects for each of the three GSTT1 and GSTM1 genotypes (i.e., homozygous-present, heterozygous, and null samples) were analyzed using ethidium bromide stained gels. All samples that had been assigned the null genotype using the TaqMan assay failed to generate a GSTT1- or GSTM1-specific PCR product despite co-amplification of the reference gene product (data not shown). The samples that were identified as heterozygote or homozygouspresent with the quantitative TaqMan assay gave similar results, each showing a GSTT1- or GSTM1-specific band as well as reference gene products. The relative intensity of bands for heterozygotes versus homozygous-present samples was not sufficiently different to distinguish between these two genotypes.

In summary, the closed-tube quantitative dual-label TaqMan PCR assay with same-well normalization to a reference gene provides a robust method for the discrimination of one versus two gene copies at the two loci examined. Only a small number of samples required re-amplification to obtain satisfactory amplification ( 10 of 270 samples for GSTT1 and 7 of 270 samples for GSTM1). Repeat analysis of 30 randomly selected samples each for the GSTT1 and GSTM1 showed a $100 \%$ concordant replication. The same assay should be useful for genotyping at other loci where there are deletions and to differentiate two from three gene copies in trisomy conditions by using an increased number of replicates to further reduce the between-sample variation in $\Delta \mathrm{C}_{\mathrm{t}}$. Compared to other techniques that have been used to assay relative genetic copy number-Southern blot analysis, competitive PCR, and fluorescent in situ hybridization (FISH) - the quantitative TaqMan assay is technically less demanding, involves less time to complete, and can be easily scaled to examine a few or hundreds of samples. We are currently exploring the use of this method to identify the ends of disease-associated deletions as part of positional cloning strategies in family-based genetic studies.

\section{ACKNOWLEDGMENTS}

This study was supported by $\mathrm{Na}$ tional Intitutes of Health grant nos. M01-RR06192 [General Clinical Research Center at the University of Connecticut Health Center (UCHC)], 2P50-AA003510-26 (Alcohol Research Center at UCHC), K24-AA13736 (to H.K.), and R01-DA15167 (to C.O.).

\section{REFERENCES}

1.Garte, S., L. Gaspari, A.K. Alexandrie, C. Ambrosone, H. Autrup, J.L. Autrup, H. Baranova, L. Bathum, et al. 2001. Metabolic gene polymorphism frequencies in control populations. Cancer Epidemiol. Biomarkers Prev. 10:1239-1248.

2.Zhong, S., A.H. Wyllie, D. Barnes, C.R. Wolf, and N.K. Spurr. 1993. Relationship between the GSTM1 genetic polymorphism and susceptibility to bladder, breast and colon cancer. Carcinogenesis 14:1821-1824.

3.Kelsey, K.T., M.R. Spitz, Z.F. Zuo, and J.K.
Wiencke. 1997. Polymorphisms in the glutathione S-transferase class mu and theta genes interact and increase susceptibility to lung cancer in minority populations (Texas, United States). Cancer Causes Control 8:554-559.

4.Taioli, E., L. Gaspari, S. Benhamou, P. Boffetta, J. Brockmoller, D. Butkiewicz, I. Cascorbi, M.L. Clapper, et al. 2003. Polymorphisms in CYP1A1, GSTM1, GSTT1 and lung cancer below the age of 45 years. Int. J. Epidemiol. 32:60-63.

5.Yuille, M., A. Condie, C. Hudson, Z. KoteJarai, E. Stone, R. Eeles, E. Matutes, D. Catovsky, et al. 2002. Relationship between glutathione S-transferase M1, T1, and P1 polymorphisms and chronic lymphocytic leukemia. Blood 99:4216-4218.

6.Duell, EJ., E.A. Holly, P.M. Bracci, M. Liu, J.K. Wiencke, and K.T. Kelsey. 2002. A population-based, case-control study of polymorphisms in carcinogen-metabolizing genes, smoking, and pancreatic adenocarcinoma risk. J. Natl. Cancer Inst. 94:297-306.

7.Wang, X., B. Zuckerman, C. Pearson, G. Kaufman, C. Chen, G. Wang, T. Niu, P.H. Wise, et al. 2002. Maternal cigarette smoking, metabolic gene polymorphism, and infant birth weight. JAMA 287:195-202.

8.Wiebel, F.A., A. Dommermuth, and R. Thier. 1999. The hereditary transmission of the glutathione transferase hGSTT1-1 conjugator phenotype in a large family. Pharmacogenetics 9:251-256.

9. Hallier, E., R. Jager, S. Deutschmann, H.M. Golt, and H. Peter. 1990. Glutathione conjugation and cytochrome P-450 metabolism of methyl chloride in vitro. Toxicol. In Vitro 4: 513-517.

10.Ingham, D., S. Beer, S. Money, and G. Hansen. 2001. Quantitative real-time PCR assay for determining transgeen copy number in transformed plants. BioTechniques 31: 132-140.

11.Holland, P.M., R.D. Abramson, R. Watson, and D.H. Gelfand. 1991. Detection of specific polymerase chain reaction product by utilizing the $5^{\prime}----3^{\prime}$ exonuclease activity of Thermus aquaticus DNA polymerase. Proc. Natl. Acad. Sci. USA 88:7276-7280.

12.Livak, K.J., S.J. Flood, J. Marmaro, W. Giusti, and K. Deetz. 1995. Oligonucleotides with fluorescent dyes at opposite ends provide a quenched probe system useful for detecting PCR product and nucleic acid hybridization. PCR Methods Appl. 4:357-362.

13.Heid, C.A., J. Stevens, K.J. Livak, and P.M. Williams. 1996. Real time quantitative PCR. Genome Res. 6:986-994.

Received 22 May 2003; accepted 26 June 2003.

Address correspondence to Jonathan Covault, Department of Psychiatry, MC 2103, UCHC, Farmington, CT 06030-2103, USA e-mail: covault@psychiatry.uchc.edu 